\title{
Masses and Widths of Resonances for the Cornell Potential
}

\author{
M. N. Sergeenko \\ Stepanov Institute of Physics, Belarus National Academy of Sciences, 68 Nezavisimosti Avenue, 220072 Minsk, Belarus
}

Correspondence should be addressed to M. N. Sergeenko; msergeen@usa.com

Received 5 April 2013; Revised 10 July 2013; Accepted 1 September 2013

Academic Editor: Terry Sloan

Copyright (C) 2013 M. N. Sergeenko. This is an open access article distributed under the Creative Commons Attribution License, which permits unrestricted use, distribution, and reproduction in any medium, provided the original work is properly cited.

Physical properties of the Cornell potential in the complex-mass scheme are investigated. Two exact asymptotic solutions of relativistic wave equation for the coulombic and linear components of the potential are used to derive the resonance complexmass formula. The centered masses and total widths of the leading $\rho$-family resonances are calculated.

\section{Introduction}

Resonance is the tendency of a system to oscillate at a greater amplitude at some frequencies. Most particles listed in the Particle Data Group tables [1] are unstable. A thorough understanding of the physics summarized by the PDG is related to the concept of a resonance.

Operators in Quantum Mechanics (QM) are Hermitian, and the corresponding eigenvalues are real. However, in scattering experiment, the wave function requires different boundary condition that is why the complex energy is required [2, 3]. The concept of a purely outgoing wave belonging to the complex eigenvalue $\mathscr{E}_{n}=E_{n}-i \Gamma_{n} / 2$ was introduced in 1939 by Siegert [4]. It is an appropriate tool in the studying of resonances. Resonance is present as transient oscillations associated with metastable states. Fundamentals of scattering theory and strict mathematical definition of resonances in QM were considered in $[5,6]$.

In elastic and inelastic scattering experiments, the resonances are associated with the scattering particle and the target [7]. Resonances can be thought of as unstable particles with the particle's complex energy poles in the scattering amplitude. In such case the energy decreases exponentially with the time, so that the damping constant $\Gamma$ is a measure of the lifetime, $\tau=h / \Gamma$, of the oscillation.

In particle physics resonances arise as unstable intermediate states with complex masses. Resonances in Quantum Field Theory (QFT) are described by the complex-mass poles of the scattering matrix [3]. In potential theory the decay widths come from second- or higher-order perturbation theory. The complex eigenvalues correspond to a first-order pole of the
$S$-matrix [8]. The origin of mass and widths of resonances in QFT originate from the real and imaginary parts of Feynman diagrams, that is, from propagators appearing in loops. The masses of the states develop imaginary masses from loop corrections $[9,10]$.

In the pole approach the parameters of resonances are defined in terms of the pole position $s_{p}$ in the complex $s$-plane as $[5,6,11,12]$

$$
s_{p}=M_{p}^{2}-i M_{p} \Gamma_{p}
$$

where $s=\left(p_{a}+p_{b}\right)^{2}=\mathscr{M}^{2}$ is the complex Mandelstam variable, $M_{p}$ is the resonance centered mass, and $\Gamma_{p}$ is the resonance width. In this approach, resonances are defined as the poles of the meromorphic continuation of the cut-off resolvent, which are shown to be the same as the poles of the meromorphically continued $S$-matrix. This definition has the advantage of being quite universal regarding the pole position but can only be applied if the amplitude can be analytically continued in a reliable way.

The complex-mass scheme provides a consistent framework for dealing with unstable particles and has been successfully applied to various loop calculations [9]. The rigorous QM definition of a resonance requires determining the pole position in the second Riemann sheet of the analytically continued partial-wave scattering amplitude in the complex Mandelstam $s$ variable plane [13]. This definition has the advantage of being quite universal regarding the pole position but can only be applied if the amplitude in scattering experiment can be analytically continued in a reliable way. 
However, there are two basic problems in quantum physics: the scattering problem and bound state problem. Resonances are usually studied in scattering experiment, for example, potential scattering. But, resonances are quasibound states in the $s$-channel at $s>0$ that means one can use another approach to consider resonances, that is, in bound state region using the quark potential model.

In perturbative QCD, the essential interaction at small distances is instantaneous coulombic one-gluon exchange (OGE); in QCD, it is 99 , 99 , or $g g$ Coulomb scattering [14]. Therefore, one expects from OGE a Coulomb-like contribution to the potential: $V_{S}(r) \propto-\alpha_{s} / r$ at $r \rightarrow 0$. For large distances, from lattice-gauge-theory computations [15], it follows that this rise is an approximately linear; that is, $V_{L}(r) \simeq \sigma r+$ const, where $\sigma \simeq 0.15 \mathrm{GeV}^{2}$ is the string tension. These two contributions by simple summation lead to the famous Cornell $q \bar{q}$ potential [15-17]:

$$
V(r)=V_{S}(r)+V_{L}(r) \equiv-\frac{4}{3} \frac{\alpha_{s}}{r}+\sigma r,
$$

and its parameters are directly related to basic physical quantities noted above. All phenomenologically acceptable QCD-inspired potentials are only variations around this potential.

The potential (2) is one of the most popular in hadron physics and incorporates in clear form the basic features of the strong interaction. In hadron physics, the nature of the potential is very important. There are normalizable solutions for scalar-like potentials, but not for vector-like. The effective interaction has to be Lorentz-scalar in order to confine quarks and gluons [18]; that is, we take the potential (2) to be Lorentzscalar.

Our aim is to find in analytic form the energy/mass eigenvalues for the potential (2). This problem is not easy if one uses known relativistic wave equations. However, this aim can be achieved with the use of the semiclassical wave equation [19]. An important feature of this equation is that, for two and more turning-point problems, it can be solved exactly by the conventional WKB method [19]. Another way is to use the complex-mass scheme.

In this work, in contrast to the usual analysis of the scattering amplitude, we consider mesonic resonances in bound state region, that is, quasibound states of the meson constituents. In traditional approach to investigate resonances, one deals with the scattering theory, exploring the properties of $S$-matrix and partial amplitudes. We consider mesonic resonances to be the transient excited states of a quarkantiquark system in bound state region. We use the potential approach and analyze the mass spectrum generated from solution of the relativistic wave equation for two components of the Cornell potential-coulombic and linear.

We use the advantage of analyzing the system in the complex plane (the complex-mass scheme) that has important features such as a simpler and more general framework $[9,10]$. We show that two asymptotic components of the Cornell potential, the coulombic term and linear one, yield the complex masses of resonances that allows to calculate in unified way their centered masses and total widths.

\section{The Complex Eigenmomenta}

Operators of physical values in conventional QM are Hermitian. However, the nature demonstrates its nonexhausting complexity. And now, we must accept the fact that energy and mass are complex. This is clearly shown in particle physics $[7,9,20]$.

It is possible to extend the Hamiltonian that describes a quantum system into the complex domain while still retaining the fundamental properties of a quantum theory. One of such approaches is Complex Quantum Mechanics (CQM) [21]. The CQM has proved to be so interesting that research activity in this area has motivated studies of complex classical mechanics [22]. Each unstable particle in CQM is associated with a well-defined object, which is a discrete generalized eigenstate of the Hamiltonian having its real and negative imaginary parts being the binding energy, $E_{n}$, and half-width, $\Gamma_{n} / 2$, of the particle, respectively $[21,22]$. Here $\Gamma_{n}$ corresponds to the inverse of its lifetime, $\Gamma_{n}=1 / \tau_{n}$, of a resonance state. The time dependence of the survival amplitude in the small-time region is strongly correlated with the asymptotic behavior of the energy spectrum of the system.

Complex energy means the complex momentum and mass. We observe an important hint studying the hydrogen atom problem. The total energy eigenvalues for the nonrelativistic Coulomb problem can be written with the use of complex quantities in the form of kinetic energy for a free particle:

$$
E_{n}=\frac{p_{n}^{2}}{2 m}, \quad p_{n}=\frac{i \alpha m}{N} .
$$

Here $p_{n}=m v_{n}$ is the electron's momentum eigenvalue with the imaginary discrete velocity, $v_{n}=i \alpha / N, N=n_{r}+l+1$. This means that the motion of the electron in a hydrogen atom is free but restricted by the "walls" of the potential [19, 23].

The Cornell potential (2) is unique in that sense, and it yields the complex masses of resonances. To show that, analyze the eigenvalues obtained separately for two components of the potential (2), that is, the coulombic term and the linear one.

Relativistic two-body Coulomb problem for particles of equal masses can be solved analytically [23]. The exact expression for the c.m. energy squared is well known and can be written in the form of two free relativistic particles as $[24,25]$

$$
E_{n}^{2}=4\left[\left(i \operatorname{Im} \pi_{n}\right)^{2}+m^{2}\right], \quad \operatorname{Im} \pi_{n}=\frac{\widetilde{\alpha} m}{N},
$$

where $N$ is given above and $\widetilde{\alpha}=(4 / 3) \alpha_{s}$. Here we have introduced the imaginary momentum eigenvalues, $i \operatorname{Im} \pi_{n}$.

The linear term of the Cornell potential (2) can be dealt with analogously. In this case the exact solution is also well known [23-25]:

$$
E_{n}^{2}=8 \sigma \widetilde{N}, \quad \widetilde{N}=N+n_{r}+\frac{1}{2} .
$$


This expression does not contain the mass term and can be written similar to (4) relativistic form:

$$
E_{n}^{2}=4\left(\operatorname{Re} \pi_{n}\right)^{2}, \quad \operatorname{Re} \pi_{n}=\sqrt{2 \sigma \widetilde{N}}
$$

where $\operatorname{Re} \pi_{n}$ is the real momentum eigenvalue.

Thus, two asymptotic additive terms of the potential (2), $V_{S}(r)$ and $V_{L}(r)$, separately, yield the imaginary (4) and the real (6) momentum eigenvalues. These terms of the potential represent two "different physics" (coulombic OGE and linear string tension) and therefore two different realms of the interaction. Each of these two expressions, (4) and (6), is exact and was obtained independently; therefore, we can consider the complex sum:

$$
\pi_{n}=\sqrt{2 \sigma \widetilde{N}}+i \frac{\widetilde{\alpha} m}{N}
$$

Thus, we accept the complex momentum eigenvalues (7) that means the total energy and mass should be complex as well.

\section{The Complex Quark Mass}

The real and imaginary parts of complex numbers/functions are independent. Particle decays are associated with complex mass. The centered mass, $M_{n}^{R}=\operatorname{Re} \mathscr{M}_{n}$, and the total width, $\Gamma_{n}^{\mathrm{TOT}}=-2 \mathrm{Im} \mathscr{M}_{n}$, are process-independent parameters of the resonance; they are the real and imaginary parts of the complex mass $\mathscr{M}_{n}$.

It is an experimental fact that the dependence $M_{n}^{2}(J)$ is linear for light mesons [20]. However, at present, the best way to reproduce the experimental masses of particles is to rescale the entire spectrum given by (5) assuming that the masses of the mesons are expressed by the relation [18]

$$
M_{n}^{2}=8 \sigma\left(2 n_{r}+l+\frac{3}{2}\right)-C^{2}
$$

where $C$ is a constant energy (shift parameter). Relation (8) is used to shift the spectra and appears as a means to simulate the effects of unknown structure approximately. But, if we rewrite (8) in the usual relativistic form,

$$
M_{n}^{2}=4\left[\left(\operatorname{Re} \pi_{n}\right)^{2}+\left( \pm i \mu_{I}\right)^{2}\right]
$$

where $\operatorname{Re} \pi_{n}$ is given by (6), we come to the concept of the imaginary mass, $\mu_{I}$. Here in (9), we have introduced the notation, $4\left( \pm i \mu_{I}\right)^{2}=-C^{2}$. What is the mass $\mu_{I}$ ?

The required shift of the spectra naturally follows from the asymptotic solution of the semiclassical wave equation for the potential (2) [23-26]. To show that, we account for the "weak coupling effect"; that is, together with the linear dependence in (6), we should include the contribution of the coulombic term, $-\alpha_{s} / r$, of the potential.

These kinds of calculations result in the asymptotic expression similar to $(8)[23,24]$ :

$$
M_{n}^{2}=8 \sigma(\widetilde{N}-\widetilde{\alpha})
$$

The additional term, $-8 \tilde{\alpha} \sigma$, arises from the interference of the coulombic and linear components of the Cornell potential (2). Comparing (10) with (9), we obtain

$$
\mu_{I}= \pm \sqrt{2 \widetilde{\alpha} \sigma}
$$

The interference term $-8 \widetilde{\alpha} \sigma$ in (10) contains only the parameters of the potential (2) and is Lorentz-scalar, that is, additive to the particle masses. This is why, we accept the last term in (10) to be the mass term, that is, (11) is the imaginarypart mass generated by the interference term of the Cornell potential (2).

Thus, we have the particle real-part (constituent) mass, $\mu_{R}=m$, and the imaginary-part mass, $\mu_{I}$, originating from the potential. As in case of the eigenmomenta, we introduce the complex mass:

$$
\mu=m-i \sqrt{2 \widetilde{\alpha} \sigma}
$$

which we use to reconstruct the complex masses of resonances, that is, their centered masses and widths.

\section{The Complex Masses of Resonances}

Resonances are complex-mass values and can be described by complex numbers. These numbers are important even if one wants to find real solutions of a problem. Using complex numbers, we are getting more than what we insert. Separate consideration of two components of the Cornell potential (2) results in the complex momentum (7) and mass (12) of bound particles. These complex expressions can be combined in one: the mass squared of two free relativistic particles with the complex momenta (7) and masses (12) as $\mathscr{M}_{n}^{2}=4\left[\left(\pi_{n}\right)^{2}+\mu^{2}\right]$; that is,

$$
\mathscr{M}_{n}^{2}=4\left[\left(\sqrt{2 \sigma \widetilde{N}}+\frac{i \widetilde{\alpha} m}{N}\right)^{2}+(m-i \sqrt{2 \widetilde{\alpha} \sigma})^{2}\right] .
$$

The complex-mass expression (13), in which the real and imaginary parts are exact, is also exact. This formula is an Anzatz like the Cornell potential (2). It can be written in another form as

$$
\mathscr{M}_{n}^{2}=\operatorname{Re} \mathscr{M}_{n}^{2}+i \operatorname{Im} \mathscr{M}_{n}^{2}
$$

where

$$
\begin{gathered}
\operatorname{Re} \mathscr{M}_{n}^{2}=4\left[2 \sigma \widetilde{N}-\left(\frac{\widetilde{\alpha} m}{N}\right)^{2}+m^{2}-\mu_{I}^{2}\right], \\
\operatorname{Im} \mathscr{M}_{n}^{2}=8 m \mu_{I}\left(\frac{\sqrt{\widetilde{\alpha} \widetilde{N}}}{N}-1\right) .
\end{gathered}
$$

The real part (15) exactly coincides with the universal mass formula obtained independently by another method with the use of the two-point Padé approximant [24, 25, 27, 28]. The simple mass formula (15) describes equally well the mass spectra of all $q \bar{q}$ and $Q \bar{Q}$ mesons ranging from the $u \bar{d}(d \bar{d}$, $u \bar{u}, s \bar{s})$ states up to the heaviest known $b \bar{b}$ systems [27, 28]. It has been used to calculate the glueball masses as well $[24,25]$. 
The universal mass formula (15) is very transparent physically, as well as the potential (2) (OGE + linear). It allows us to get an analytic expression for Regge trajectories in the whole region. Transforming (15) we obtain the cubic equation for the angular momentum $J\left(s=\mathscr{M}^{2}\right)$ [24]:

$$
J^{3}+a_{1}(s) J^{2}+a_{2}(s) J+a_{3}(s)=0 .
$$

Solution obtained from (17) results in the "saturating" Regge trajectories including the Pomeron [24, 25] and appears to be successful in many applications.

The "saturating" Regge trajectories (see Figure 1) [27, 28] were applied with success to the photoproduction of vector mesons that provide an excellent simultaneous description of the high and low $-t$ behavior of the $\gamma p \rightarrow p, \rho, \omega, \Phi$ cross-sections $[33,34]$, given an appropriate choice of the relevant coupling constants (JML-model) [35, 36]. It was shown that the hard-scattering mechanism is incorporated in an effective way by using the "saturated" Regge trajectories that are independent of $t$ at large momentum transfers [27, 28].

Saturating trajectories have a close phenomenological connection to the quark-antiquark interaction which governs the mesonic structure $[25,27,28]$. They provide an effective way to implement gluon exchange between the quarks forming the exchanged meson $[33,37]$ and lead to the asymptotic quark counting rules [38] that, model independently, determine the energy behavior of the cross section at large $-t$. This approach was successfully adopted to explain the large momentum transfer hadron-hadron interactions, as well as several photon-induced reactions [39]. The pion saturating trajectory $\left(\alpha_{\pi}^{\text {sat }}(s)=-1\right.$ when $\left.t \rightarrow-\infty\right)$ is in a form that reproduces the $\gamma p \rightarrow n \pi^{+}$reaction around $\theta_{\omega}^{*}=90$ [39].

In general (mathematically), the $S$-matrix is a meromorphic function of complex variable $\mathscr{M}= \pm \sqrt{s}$, where the complex $s$-plane is replaced by the two-sheet Riemann surface, $\pm \sqrt{s}$, made up of two sheets $R_{0}$ and $R_{1}$, each cut along the positive real axis, $\operatorname{Re} \mathscr{M}$, and with $R_{1}$ placed in front of $R_{0}$ $[5,13]$. The square root of the complex expression (14) gives

$$
\sqrt{\mathscr{M}_{n}^{2}}=\operatorname{Re} \mathscr{M}_{n}+i \xi \operatorname{Im} \mathscr{M}_{n}
$$

where

$$
\begin{aligned}
& \operatorname{Re} \mathscr{M}_{n}= \pm \sqrt{\frac{\left|\mathscr{M}_{n}^{2}\right|+\operatorname{Re} \mathscr{M}_{n}^{2}}{2}} \\
& \operatorname{Im} \mathscr{M}_{n}= \pm \sqrt{\frac{\left|\mathscr{M}_{n}^{2}\right|-\operatorname{Re} \mathscr{M}_{n}^{2}}{2}}
\end{aligned}
$$

where $\left|\mathscr{M}_{n}^{2}\right|=\left[\left(\operatorname{Re} \mathscr{M}_{n}^{2}\right)^{2}+\left(\operatorname{Im} \mathscr{M}_{n}^{2}\right)^{2}\right]^{1 / 2}$ and $\xi=$ $\operatorname{sgn}\left(\operatorname{Im} \mathscr{M}_{n}^{2}\right)$.

The expressions (19) and (20) define the resonance positions which are symmetrically located in the Riemann $\mathscr{M}$ surface. Resonance masses arise in complex-conjugate pairs. Poles in the left half-plane correspond to either bound or antibound states $[2,40]$. If $\mathscr{M}_{n}=M_{n}-i \Gamma_{n} / 2$ is a pole in the fourth quadrant of the surface $\pm \sqrt{s}$, then $\mathscr{M}_{n}=-M_{n}-i \Gamma_{n} / 2$ is also a pole, but in the third quadrant (antiparticle) [2].

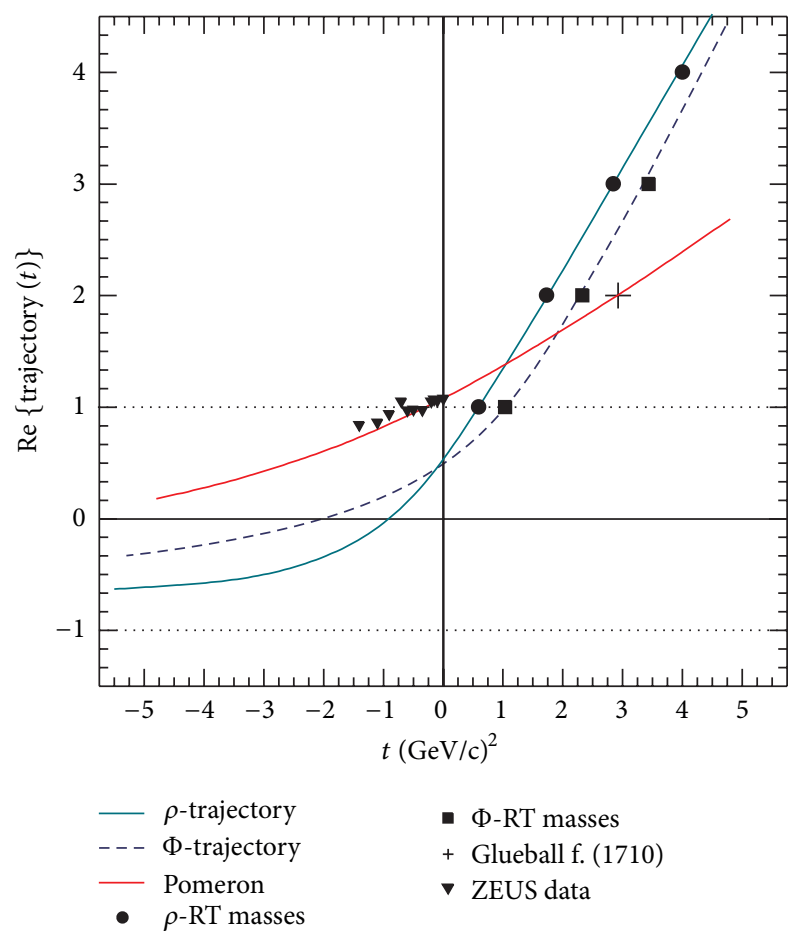

Figure 1: The Chew-Frautschi plots of the leading $\rho, \Phi$, and $P$ complex Regge trajectories calculated from (17) [24, 25]. Parameters are found from the fit of combined HERA $\rho$ and $\Phi$ data (triangles) [29-31] and $2^{++}$glueball candidate $f_{0}(1710)$ (cross) [32]. Solid curve is the $P$ trajectory; dotted and dashed lines show the leading $\rho$ and $\Phi$ saturating Regge trajectories.

As mentioned above, the masses of the states develop imaginary masses from loop corrections. In this case, the probability density comes from the particle's propagator, with the complex mass, $\mathscr{M}_{n}=M_{n}-i \Gamma_{n} / 2$. This formula is related to the particle's decay rate by the optical theorem $[3,40]$. That means the total widths are

$$
\Gamma_{n}^{\mathrm{TOT}}=-2 \operatorname{Im} \mathscr{M}_{n}
$$

where the imaginary mass is given by (20). The centered mass, $M_{n}^{R}=\operatorname{Re} \mathscr{M}_{n}$, and the total width, $\Gamma_{n}^{\mathrm{TOT}}$, are processindependent parameters of the resonance.

There is the lack of a precise definition of what is meant by mass and width of resonance. Comprehensive definitions of these resonance's parameters require further investigations. An alternate definition of the resonance's width can be obtained from (16). According to definition (21), the width is given by the negative imaginary-part mass of the resonance's complex mass, $\mathscr{M}_{n}$.

In the pole approach, the pole position $s_{p}$ in the complex $s$-plane is given by (1). Comparing (14) with (1), we obtain the equality

$$
-M_{p} \Gamma_{p}=8 m \mu_{I}\left(\frac{\sqrt{\tilde{\alpha} \widetilde{N}}}{N}-1\right) .
$$


TABLE 1: Masses and total widths of the $\rho$-family resonances.

\begin{tabular}{|c|c|c|c|c|c|}
\hline Meson & $M_{n}^{\mathrm{ex}}$ & $M_{n}^{\text {th }}$ & $\Gamma_{n}^{\mathrm{ex}}$ & $\Gamma_{n}^{\mathrm{th}}(21)$ & $\Gamma_{n}^{\mathrm{th}}(24)$ \\
\hline$\rho(1 S)$ & 776 & 775 & 149 & 150 & 75 \\
\hline$a_{2}(1 P)$ & 1318 & 1323 & 107 & 108 & 93 \\
\hline$\rho_{3}(1 D)$ & 1689 & 1689 & 161 & 170 & 188 \\
\hline$a_{4}(1 F)$ & 1996 & 1985 & 255 & 194 & 249 \\
\hline$\rho_{5}(1 G)$ & - & 2234 & - & 202 & 294 \\
\hline$a_{6}(1 H)$ & - & 2462 & - & 205 & 328 \\
\hline
\end{tabular}

The question arises: how should we treat (22)? One choice is

$$
\Gamma_{n}^{\mathrm{TOT}} \equiv \Gamma_{p}=\frac{8 m}{M_{n}}\left|\mu_{I}\left(\frac{\sqrt{\tilde{\alpha} \widetilde{N}}}{N}-1\right)\right| .
$$

An alternate definition of the resonance's width can be obtained as follows. According to (21), the width is given by the imaginary-part mass of the resonance's complex mass, $\mathscr{M}_{n}$. The mass and width in (22) are in factorized form (system mass $) \times($ width $)$; that is, $-M_{p} \Gamma_{p}=(4 m) 2 \mu_{I}(\sqrt{\tilde{\alpha} \widetilde{N}} / N-$ $1)$. Therefore, omitting the real-mass factor $(4 m)$, one can consider the following definition:

$$
\Gamma_{n}^{\mathrm{TOT}}=-2 \operatorname{Im} \mathscr{M}_{n}=2 \mu_{I}\left(\frac{\sqrt{\widetilde{\alpha} \widetilde{N}}}{N}-1\right) .
$$

This value depends on the imaginary-part mass $\mu_{I}$ of complex mass (12) and quantum numbers. It has the maximum value given by the imaginary mass, $\mu_{I}=-\sqrt{2 \widetilde{\alpha} \sigma}$,

$$
\Gamma_{n}^{\max }=-2 \mu_{I}=2 \sqrt{2 \tilde{\alpha} \sigma} .
$$

As an example, consider the $\rho$-family resonances of the leading Regge trajectory, $\alpha_{\rho}(s)$. Calculations are given in Table 1, where masses and widths are in $\mathrm{MeV}$. Note that expressions (20) and (23) give very close results, for $\Gamma_{n}^{\mathrm{TOT}}$. Centered masses were calculated with the use of (19).

Parameters values in these calculations are found from the best fit to the available data [1]: $\alpha_{s}=1.463, \sigma=$ $0.134 \mathrm{GeV}^{2}$, and $m=193 \mathrm{MeV}$. The widths $\Gamma_{n}^{\text {th }}(20)$ and $\Gamma_{n}^{\text {th }}(24)$ are calculated with the use of (20) and (24). The maximum imaginary mass is $\mu_{I}=723 \mathrm{MeV}$. The spin-dependent corrections to the potential (2) have been considered in [28]. Location of the $\rho$-family resonances in the complex $\mathscr{M}$ surface is shown in Figure 2.

\section{Conclusion}

A thorough understanding of the physics summarized by the PDG is related to the concept of a resonance. Resonances in hadron physics are never observed directly but through their decay channels that causes problems with their definition. In addition to known definitions of what is meant by mass and width of resonance, we have considered the alternate definition (24).

In contrast to the usual analysis dealing with the scattering problem, we have modeled mesonic resonances to be

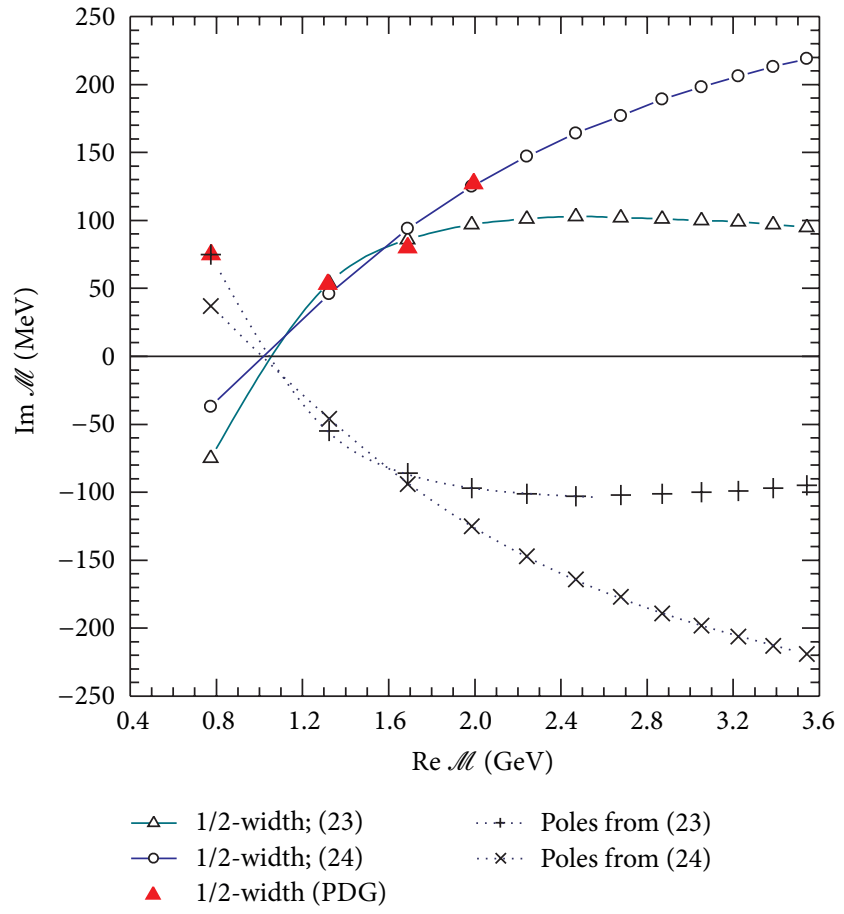

FIgURE 2: The complex-conjugate poles in the Riemann $\mathscr{M}=$ $\pm \sqrt{s}$-surface for the $\rho$ family resonances. Stars show location of the complex-mass resonances on the leading $\rho$ Regge trajectory; the imaginary-part component is the resonance half-width. Crosses show calculation results with the use of (20) and (24).

the quasibound eigenstates of two interacting quarks. We have chosen the Cornell potential to describe the interquark interaction. Using the complex-mass scheme, we have analyzed the exact eigenvalues for the coulombic and linear terms of the potential, separately, and obtained the complex-mass formula for meson resonances. This approach has allowed us to simultaneously describe in the unified way the masses and total widths of resonances in a good agreement with data.

There exists a widely spread belief that the width of heavy hadronic resonances linearly depends on their mass. We have found this inconsistent with an existence of linear Regge trajectories. In our analysis the total width of resonances $\Gamma_{n}^{\mathrm{TOT}}$ is finite or decreasing; according to (25), it may have the maximum value, which equals to imaginary-part mass, $\mu_{I}$. The complex-mass expression (13) may have relation to some nonhermitian Hamiltonian. 
Note a feature of the $\rho$-family resonance data. There is a dip $\left(\Gamma_{n}^{\mathrm{TOT}}=107 \mathrm{MeV}\right.$ ) for the $a_{2}(1320)$ resonance (see Figure 2). This dip is described in our scheme and may have the following explanation. It is known that complex poles of the $S$-matrix always arise in conjugate pairs $[40,41]$. Poles in the lower half-plane are complex-conjugated with zeros in upper half-plane. Zeros above the real axis correspond to bound states, and resonances are located in the lower halfplane.

According to (18), the imaginary-part mass $\operatorname{Im} \mathscr{M}_{n}$ can be positive and negative, poles can be located above and below the real $\mathscr{M}$-axis in the complex $\mathscr{M}$-surface; that is, in bound state or scattering region. This choice depends on boundary conditions. Analysis and numerical calculations using (18)(24) show that the imaginary-part mass $\operatorname{Im} \mathscr{M}_{n}$ of the $\rho(770)$ resonance is positive; that is, the $\rho(770)$-state is located in the first quadrant of the complex $\mathscr{M}$-surface $(\operatorname{Re} \mathscr{M}>0, \operatorname{Im} \mathscr{M}>$ $0)$. All other states of the $\rho(770)$-family Regge trajectory are embedded in the fourth quadrant $(\operatorname{Re} \mathscr{M}>0, \operatorname{Im} \mathscr{M}<0)$. What does it mean?

Explanation to this phenomenon may be as follows. If the $\rho$ (770)-state is located in the first quadrant, it is a quasibound state, which has the zero quantum numbers, that is, $l=n_{r}=0$ (ground state). If we define resonance to be an excited state with nonzero quantum numbers, either $l$ or $n_{r}$, then $\rho$-meson is not a resonance but the quasibound state with parallel spins of quarks. This is only difference between $\rho$ and $\pi$-meson, that is, quark spin orientation. That means that in this sense all $\left(n_{r}+1\right) L\left(J^{P C}\right)=1 S\left(1^{--}\right)$-states are mesons, that is, unstable bound states with positive imaginary-part mass.

Resonances represent a very economical way in theoretical description of hadronic reactions at high energies. Such a task is very important nowadays since a great significance of the width of heavy resonances. Our analysis may be important for further development of the string model of hadrons and for improvement of such transport codes as the hadron string dynamics by including the finite width of heavy resonances. The universal complex-mass formula (13) gives a possibility to calculate and predict masses and width for hadrons/resonances listed in the Particle Data Group tables. This is important for experiments at LHC and others.

\section{Acknowledgments}

The author thanks Yu. A. Kurochkin for support and constant interest in this work. This work was done in the framework of investigations for the experiment ATLAS (LHC), code 02-0-1081-2009/2013, "physical explorations at LHC" (JINRATLAS).

\section{References}

[1] J. Beringer, "Review of particle physics," Physical Review D, vol. 86, no. 1, Article ID 010001, 2012.

[2] N. Moiseyev, "Quantum theory of resonances: calculating energies, widths and cross-sections by complex scaling," Physics Reports, vol. 302, no. 5-6, pp. 211-293, 1998.

[3] J. R. Taylor, The Quantum Theory of Nonrelativistic Collisions, Dover Publications, 2006.
[4] A. J. F. Siegert, "On the derivation of the dispersion formula for nuclear reactions," Physical Review, vol. 56, no. 8, p. 750, 1939.

[5] P. D. Hislop and C. Villegas-Blas, "Semiclassical Szego limit of resonance clusters for the hydrogen atom Stark Hamiltonian," http://arxiv.org/abs/1104.4466.

[6] R. Brummelhuis and A. Uribe, "A semi-classical trace formula for Schrödinger operators," Communications in Mathematical Physics, vol. 136, no. 3, pp. 567-584, 1991.

[7] G. Rupp, S. Coito, and E. Beveren, "Meson spectroscopy: too much excitement and too few excitations," Acta Physica Polonica $B$, vol. 5, supplement 3, pp. 1007-1014, 2012.

[8] W. Heitler and N. Hu, "Interpretation of complex roots of the s-matrix," Nature, vol. 159, no. 4049, pp. 776-777, 1947.

[9] T. Bauer, D. Djukanovic, J. Gegelia, S. Scherer, and L. Tiator, "Complex-mass scheme and resonances in EFT," in Proceedings of the 8th International Workshop on the Physics of Excited Nucleons (NSTAR '11), vol. 1432 of AIP Conference Proceedings, Reading, Mass, USA, 2011.

[10] A. Denner, S. Dittmaier, M. Roth, and D. Wackeroth, "Predictions for all processes $\mathrm{e}^{+} \mathrm{e}^{-} \rightarrow$ fermions $+\gamma$," Nuclear Physics B, vol. 560, no. 1-3, pp. 33-65, 1999.

[11] A. Bernicha, G. L. Castro, and J. Pestieau, "Pion-proton scattering and isospin breaking in the $\Delta^{0}-\Delta^{++}$system," Nuclear Physics A, vol. 597, no. 4, pp. 623-635, 1996.

[12] D. Morgan and M. R. Pennington, "Velocity dependence of absolute cross sections for charge capture by $\mathrm{Ar}^{7+}$ from groundstate and excited-state sodium," Physical Review A, vol. 48, no. 2, pp. 1185-1188, 1993.

[13] J. Nieves and E. R. Arriola, "Meson resonances at large NC: complex poles vs Breit-Wigner masses," Physics Letters B, vol. 679, no. 5, pp. 449-453, 2009.

[14] J. D. Bjorken and E. A. Paschos, "Inelastic electron-proton and $\gamma$-proton scattering and the structure of the nucleon," Physical Review, vol. 185, no. 5, pp. 1975-1982, 1969.

[15] G. S. Bali, "QCD forces and heavy quark bound states," Physics Reports, vol. 343, no. 1-2, pp. 1-136, 2001.

[16] N. Brambilla, A. Pineda, J. Soto, and A. Vairo, "Effective-field theories for heavy quarkonium," Reviews of Modern Physics, vol. 77, no. 4, pp. 1423-1496, 2005.

[17] H. M. E. Eichten, S. Godfrey, and J. L. Rosner, "Quarkonia and their transitions," Reviews of Modern Physics, vol. 80, no. 3, pp. 1161-1193, 2008.

[18] J. Sucher, "Confinement in relativistic potential models," Physical Review D, vol. 51, no. 10, pp. 5965-5966, 1995.

[19] M. N. Sergeenko, "Semiclassical wave equation and exactness of the WKB method," Physical Review A, vol. 53, no. 6, pp. 37983804,1996

[20] P. D. B. Collins, An Introduction to Regge Theory \& High Energy Physics, Cambridge University Press, Cambridge, Mass, USA, 1977.

[21] C. M. Bender, D. C. Brody, and D. W. Hook, "Quantum effects in classical systems having complex energy," Journal of Physics A, vol. 41, no. 35, Article ID 352003, 15 pages, 2008.

[22] C. D. P. Dorey and R. Tateo, "The ODE/IM correspondence," Journal of Physics A, vol. 40, no. 32, p. R205, 2007.

[23] M. N. Sergeenko, "Relativistic semiclassical wave equation and its solution," Modern Physics Letters A, vol. 12, no. 37, pp. 28592871, 1997.

[24] M. N. Sergeenko, "Glueball masses and Regge trajectories for the QCD-inspired potential," The European Physical Journal C, vol. 72, article 2128, 2012. 
[25] M. N. Sergeenko, "Glueballs and the pomeron," Europhysics Letters, vol. 89, no. 1, p. 11001, 2010.

[26] M. N. Sergeenko, "Classical solution of the wave equation," International Journal of Modern Physics A, vol. 18, no. 17, p. 1, 2003.

[27] M. N. Sergeenko, "Some properties of Regge trajectories of heavy quarkonia," Physics of Atomic Nuclei, vol. 56, pp. 365-371, 1993.

[28] M. N. Sergeenko, "An interpolating mass formula and Regge trajectories for light and heavy quarkonia," Zeitschrift für Physik C, vol. 64, no. 2, pp. 315-322, 1994.

[29] D. Aston, M. Atkinson, R. Bailey et al., "Photoproduction of $\varrho^{0}$ and $\omega$ on hydrogen at photon energies of 20 to $70 \mathrm{GeV}$," Nuclear Physics B, vol. 209, no. 1, pp. 56-76, 1982.

[30] J. Breitweg, M. Derrick, D. Krakauer et al., "Measurement of the diffractive structure function $\mathrm{F}_{2}^{D(4)}$ at HERA," The European Physical Journal C, vol. 1, no. 1-2, pp. 81-96, 1998.

[31] T. Ahmed, V. Andreev, B. Andrieu et al., "Total photoproduction cross section measurement at HERA energies," Physics Letters B, vol. 299, no. 3-4, pp. 374-384, 1993.

[32] A. Kirk, "New effects observed in central production by experiment WA102 at the CERN Omega Spectrometer," Nuclear Physics A, vol. 663, pp. 608-612, 2000.

[33] J. M. Laget, "Space-time structure of hard-scattering processes," Physical Review D, vol. 70, no. 5, Article ID 054023, 2004.

[34] M. Battaglieri, M. Brunoldi, R. de Vita et al., "Photoproduction of the omega meson on the proton at large momentum transfer," Physical Review Letters, vol. 90, no. 2, Article ID 022002, 2003.

[35] M. Battaglieri, E. Anciant, M. Anghinolfi et al., "Photoproduction of the $\rho^{0}$ meson on the proton at large momentum transfer," Physical Review Letters, vol. 87, no. 17, Article ID 172002, 5 pages, 2001.

[36] L. Morand, D. Doré, M. Garçon et al., "Deeply virtual and exclusive electroproduction of $\omega$-mesons," The European Physical Journal A, vol. 24, no. 3, pp. 445-458, 2005.

[37] F. Cano and J. M. Laget, "Compton scattering, vector-meson photoproduction, and the partonic structure of the nucleon," Physical Review D, vol. 65, no. 7, Article ID 074022, 4 pages, 2002.

[38] S. J. Brodsky and G. R. Farrar, "Scaling laws at large transverse momentum," Physical Review Letters, vol. 31, no. 18, pp. 11531156, 1973.

[39] M. Guidal, J.-M. Laget, and M. Vanderhaeghen, "Pion and kaon photoproduction at high energies: forward and intermediate angles," Nuclear Physics A, vol. 627, no. 4, pp. 645-678, 1997.

[40] L. D. Landau and E. M. Lifshitz, Quantum Mechanics, Pergamon, 1965.

[41] N. Fernández-García and O. Rosas-Ortiz, "Gamow-Siegert functions and Darboux-deformed short range potentials," Annals of Physics, vol. 323, no. 6, pp. 1397-1414, 2008. 

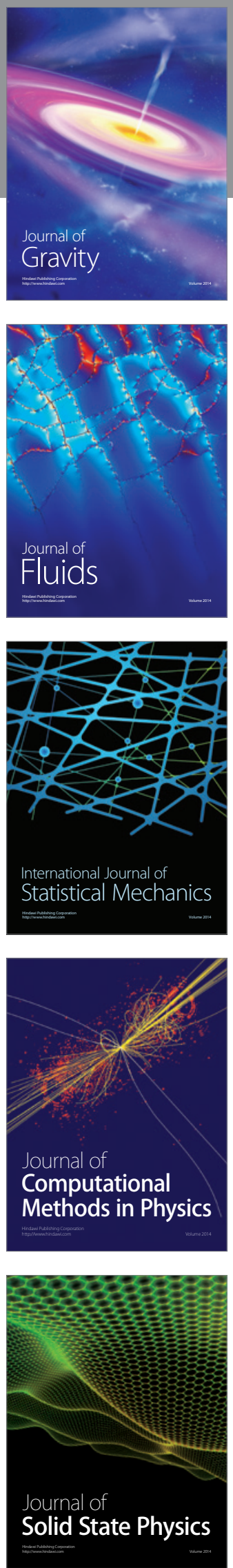

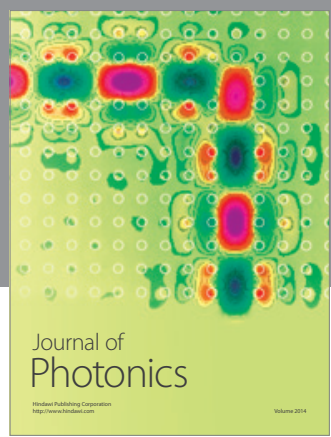

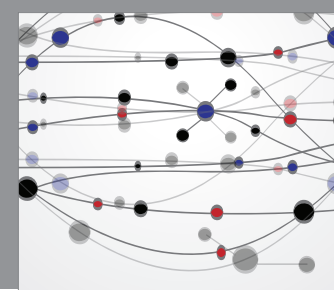

The Scientific World Journal

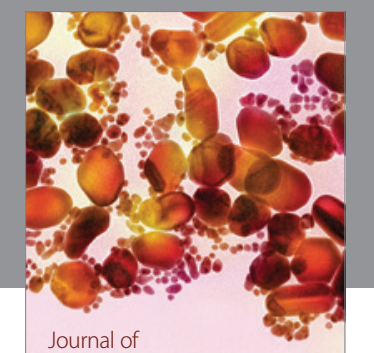

Soft Matter
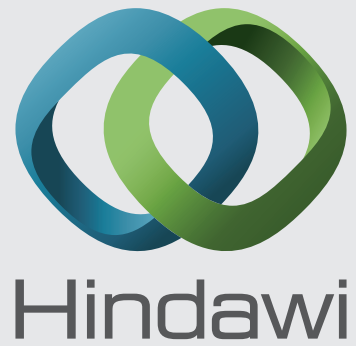

Submit your manuscripts at

http://www.hindawi.com
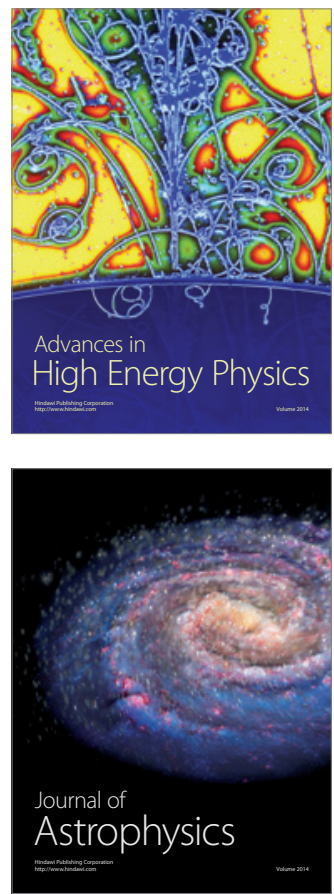
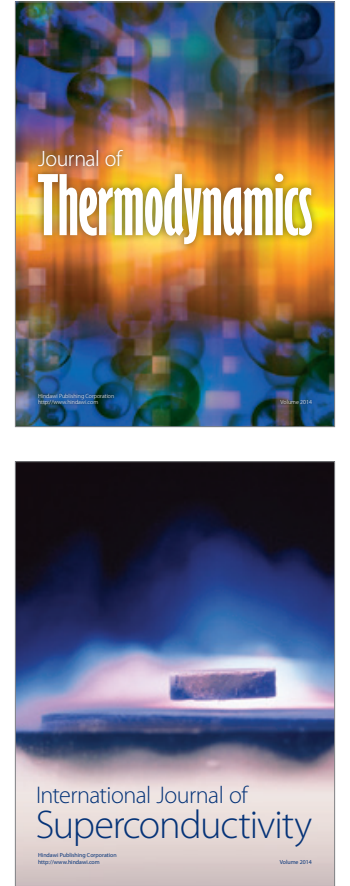
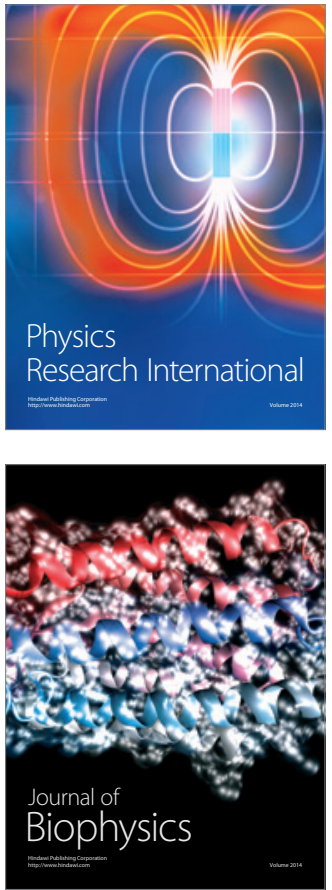
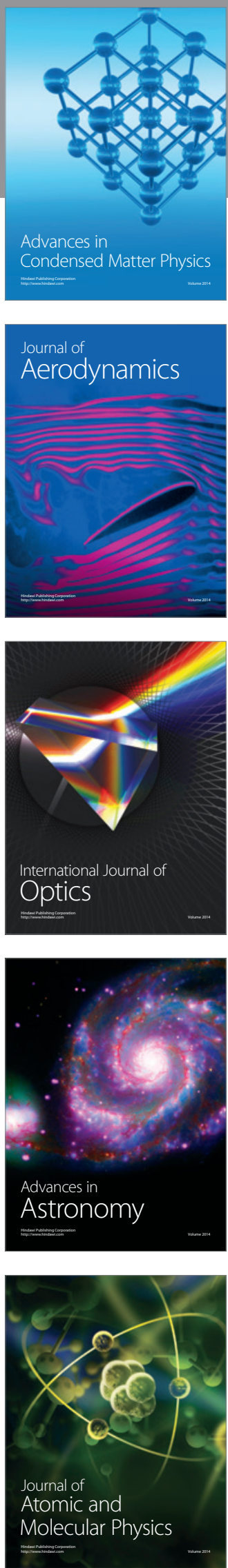